\author{
ANNALES \\ POLONICI MATHEMATICI \\ XXXIII (1977)
}

\title{
Admissible initial operators for superpositions of right invertible operators
}

\author{
by D. Przeworska-Rolewicz (Warszawa)
}

\begin{abstract}
Suppose that $D$ is a linear right invertible operator acting in a linear space $X$ and that $F$ is an initial operator for $D$ corresponding to a right inverse $R$ of $D$. Then the set of all right inverses of $D$ is of the form: $\mathscr{R}_{D}=\left\{R+F A: A \in L_{0}(X)\right\}$, where $L_{0}(X)$ denotes the space of all linear operators determined on the whole space $X$ and mapping $X$ into itself. The set of all initial operators for $D$ is of the form: $\mathscr{F}_{D}$ $=\left\{F\left(I-A D: A \in L_{0}(X)\right\}\right.$. Having already this characterization of the sets $\mathscr{R}_{D}$ and $\mathscr{F}_{D}$, we can desoribe all admissible initial operators for a superposition of a finite number of right invertible operators. A generalization of a theorem about the existence and uniqueness of a solution of an initial value problem with a right invertible operator is also given. Applications to multi-waves equations are indicated (even in the case of variable speed of waves).
\end{abstract}

In the present paper we determine the class of all initial operators for a superposition of given right invertible operators (cf. [2]). The problem in question was suggested to the author by Professor S. Kaliski.

This paper contains some generalizations of the results given in [2] and [5].

Let $\mathfrak{X}$ be an arbitrary ring with a unit $e$. The following characterization of right inverses is due to Arens [1]:

Suppose that $t \in \mathfrak{X}$ has a right inverse 8 . Then any right inverse of $t$ is of the form:

$$
\hat{s}=a+s(e-t a) \quad \text { for every } a \epsilon \mathfrak{X} .
$$

This characterization is also true in an arbitrary pararing with units (cf. [3]) if we consider all $a \in \mathfrak{X}$ such that the product $t a$ is well-determined. In particular, we obtain the following characterization of right inverses of right invertible linear operators:

Let $X$ be a linear space over a field $\mathscr{F}$ of scalars. Denote by $L(X)$ the set of all linear operators defined on linear subsets $\mathscr{D}_{A}$ of $X$, called the domain of $X$ and mapping $\mathscr{D}_{A}$ into $X{ }^{(1)}$.

(1) It is a pararing with units (moreover, it is a para-algebra).

8 - Annales Polonicl Mathematicl XXXmi.3 
Denote by $\boldsymbol{R}(X)$ the set of all right invertible operators belonging to $L(X)$. The set of all right inverses of an operator $D \in R(X)$ will be denoted by $\mathscr{R}_{D}$. As in [2], we assume here that $\mathscr{D}_{R}=X, \mathscr{D}_{D} \subset R X$ for $R \epsilon \mathscr{R}_{D}$. Write: $L_{0}(X)=\left\{A \in L(X): \mathscr{D}_{A}=X\right\}$.

Let $D \in \boldsymbol{R}(X)$ and $R \in \mathscr{R}_{D}$. Then every right inverse of $D$ is of the form

$$
\hat{R}=A+R(I-D A) \quad \text { for an } A \in L_{0}(X),
$$

where $I$ denotes the identity operator.

Let $\mathscr{F}_{D}$ denote the family of all initial operators for an operator $D \in \boldsymbol{R}(X)$. We recall (cf. [2]) that $F \in L(X)$ is an initial operator for $D \in R(X)$ corresponding to an $R \in \mathscr{R}_{D}$ if it is a projection onto the kernel of $D$, i.e., if $F^{2}=F, F X=Z_{D}$, where $Z_{D}=\left\{x \in \mathscr{D}_{D}: D x=0\right\}\left({ }^{2}\right)$ and, moreover, $F R=0$. By Theorem 2.2 of [2] an operator $F$ belongs to $\mathscr{F}_{D}$ if and only if

$$
F=I-R D \quad \text { on } \mathscr{D}_{D} \quad \text { for an } R \epsilon \mathscr{R}_{D} \text {. }
$$

An initial operator $\hat{F}$ corresponding to a right inverse $\hat{R}$ defined by formula (1.2) is of the form

$$
\hat{F}=F(I-A D), \quad \text { where } A \epsilon L_{0}(X),
$$

and $F$ is defined by formula (3).

Indeed, formula (3) implies that on the domain $\mathscr{D}_{D}$ we have

$$
\begin{aligned}
\hat{\boldsymbol{F}} & =I-\hat{R} D=I-[A+R(I-D A)] D \\
& =I-A D-R(I-D A) D=I-A D-R D+(R D)(A D) \\
& =(I-R D)(I-A D)=F(I-A D) .
\end{aligned}
$$

Proposition 1. Let $D \in R(X)$. Then $\hat{R} \in \mathscr{R}_{D}$ if and only if there is an $R \in \mathscr{R}_{D}$ and an $A \epsilon L_{0}(X)$ such that

$$
\hat{\boldsymbol{R}}=\boldsymbol{R}+\boldsymbol{F} \boldsymbol{A}
$$

where $F$ is an initial operator for $D$ corresponding to $R$. Moreover, an initial operator $\hat{F}$ corresponding to $\hat{\boldsymbol{R}}$ is

$$
\hat{\boldsymbol{F}}=\boldsymbol{F}(I-A D) \text {. }
$$

Proof. Sufficienry. Suppose that $R \in \mathscr{R}_{D}$ and $A \in L_{0}(X)$ are arbitrary. Write: $\hat{R}=R+F A$. Observe that

$$
\hat{R}=R+F A=R+(I-R D) A=R+A-R D A=A+R(I-D A),
$$

as in Formula (2). Since $D F=0$, we have, by definition,

$$
D \hat{R}=D(R+F A)=D R+D F A=I .
$$

(2) The kernel $Z_{D}$ is said to be the space of constants for the operator $D$. 
Hence $\hat{R} \in \mathscr{R}_{D}$. The initial operator $\hat{F}$ corresponding to $\hat{R}$ is, by definition,

$$
\begin{aligned}
\hat{\boldsymbol{F}} & =I-\hat{R} D=I-(R+F A) D=I-R D-F A D \\
& =F-F A D=F(I-A D) .
\end{aligned}
$$

Necessity. Let $\hat{R} \in \mathscr{R}_{D}$ be given and let $R \in \mathscr{R}_{D}$ be arbitrary chosen. Write

$$
F=I-\hat{R} D, \quad \hat{F}=I-\hat{R} D \quad \text { on } \mathscr{D}_{D} \quad \text { and } \quad A=R-\hat{R} \epsilon L_{0}(X) .
$$

Since $D R=I$ and $F R=0$, we conclude that

$$
\begin{aligned}
R+F^{\prime} A & =R+F(R-\hat{R})=R+F R-F \hat{R}=R-F \hat{R} \\
& =R+(I-R D) \hat{R}=R+\hat{R}-R(D \hat{R})=R+\hat{R}-R=\hat{R} .
\end{aligned}
$$

Thus $\hat{R} \in \mathscr{R}_{D}$ is of the required form. Formula (4) implies that $\hat{F}=F(I-A D)$, . which was to be proved.

An immediate consequence of Proposition 1 is

ThEOREM 2. Let $D \in \boldsymbol{R}(X)$. If $F \in L(X)$ is an arbitrary initial operator for $D$ corresponding to an $R \in \mathscr{R}_{D}$, then

$$
\begin{gathered}
\mathscr{R}_{D}=\left\{R+F A: A \epsilon L_{0}(X)\right\}, \\
\dot{\mathscr{F}}_{D}=\left\{F(I-A D): A \epsilon L_{0}(X)\right\} .
\end{gathered}
$$

A similar characterization can be given for left invertible operators (cf. [4]).

THEOREM 3. Suppose that $D_{1}, \ldots, D_{m} \epsilon \boldsymbol{R}(X)$ and that $F_{j}$ is an initial operator for $D_{j}$ corresponding to a right inverse $R_{j} \in \mathscr{R}_{D_{j}}(j=1, \ldots, m)$. Write

$$
\begin{gathered}
D=D_{1} \ldots D_{m} ; \quad R=R_{m} \ldots R_{1} \\
F=F_{m}+R_{m} F_{m-1} D_{m}+\ldots+R_{m} \ldots R_{2} F_{1} D_{2} \ldots D_{m} .
\end{gathered}
$$

Then $D \in \boldsymbol{R}(X), R \epsilon \mathscr{R}_{D}, F$ is an initial operator for $D$ corresponding to $R$ and, moreover,

$$
\begin{aligned}
& \mathscr{R}_{D}=\left\{R+F A: A \epsilon L_{0}(X)\right\}, \\
& \mathscr{F}_{D}=\left\{F(I-A D): A \epsilon L_{0}(X)\right\} .
\end{aligned}
$$

Proof. Since, by our assumption, $D_{j} R_{j}=I$ for $j=1, \ldots, m$, we conclude that

$$
\begin{aligned}
D R=D_{1} \ldots D_{m-1} D_{m} R_{m} R_{m-1} \ldots R_{1}=D_{1} \ldots D_{m-1} R_{m-1} \ldots R_{1} & =\ldots \\
\ldots=D_{1} R_{1} & =I .
\end{aligned}
$$


Thus $D \in R(X)$ and $R \epsilon \mathscr{R}_{D}$. Formula (3) implies that on $\mathscr{D}_{D}$ we have

$$
\begin{aligned}
& F= F_{m}+R_{m} F_{m-1} D_{m}+\ldots+R_{m} \ldots R_{2} F_{1} D_{2} \ldots D_{m} \\
&=I-R_{m} D_{m}+R_{m}\left(I-R_{m-1} D_{m-1}\right) D_{m}+\ldots++R_{m} \ldots R_{2}\left(I-R_{1} D_{1}\right) D_{2} \ldots D_{m} \\
& \quad+D_{m-1} D_{m}+\ldots+ \\
&=I-R_{m} D_{m}+R_{m} D_{m}-R_{m} R_{m-1} D_{m-1} \quad+R_{m} \ldots R_{2} D_{2} \ldots D_{m}-R_{m} \ldots R_{2} R_{1} D_{1} D_{2} \ldots D_{m} \\
&=I-R_{m} \ldots R_{1} D_{1} \ldots D_{m}=I-R D .
\end{aligned}
$$

Hence $F$ is an initial operator for $D$ corresponding to $R$. This and Theorem 2 together imply that the sets $\mathscr{R}_{D}$ and $\mathscr{F}_{D}$ are of the form (11) and (12) respectively.

Theorems 2 and 3 together imply another characterization of initial operators for a superposition of right invertible operators.

THEOREM 4. Suppose that all the assumptions of Theorem 3 are satisfied and that $D$ is defined by Formula (9). Write:

$$
\begin{gathered}
R=\left(R_{m}+F_{m} A_{m}\right) \ldots\left(R_{1}+F_{1} A_{1}\right), \\
F=F_{m}\left(I-A_{m} D_{m}\right)+\ldots+ \\
+\left(R_{m}+F_{m} A_{m}\right) \ldots\left(R_{2}+F_{2} A_{2}\right) F_{1}\left(I-A_{1} D_{1}\right) D_{2} \ldots D_{m},
\end{gathered}
$$

where $A_{1}, \ldots, A_{m} \in L_{0}(X)$ are arbitrary.

Then $R \epsilon \mathscr{R}_{D}$ and $F$ is an initial operator for $D$ corresponding to $R$. Moreover,

$$
\begin{aligned}
& \mathscr{R}_{D}=\left\{\left(R_{m}+F_{m} A_{m}\right) \ldots\left(R_{1}+F_{1} A_{1}\right): A_{1}, \ldots, A_{m} \in L_{0}(X)\right\} \\
& \mathscr{F}_{D}=\left\{F_{m}\left(I-A_{m} D_{m}\right)+\ldots+R_{m}+F_{m} A_{m} \ldots\right. \\
&\left.\ldots\left(R_{2}+F_{2} A_{2} F_{1}\right)\left(I-A_{1} D_{1}\right) D_{2} \ldots D_{m}: A_{1}, \ldots, A_{m} \in L_{0}(X)\right\} .
\end{aligned}
$$

Indeed, Theorem 2 implies that every operator of the form $F_{j}\left(I-A_{j} D_{j}\right)$, where $A_{j} \in L_{0}(X)(j=1, \ldots, m)$ is an initial operator for $D_{j}$ corresponding to the right inverse $R_{j}+F_{j} A$ of $D_{j}$. This and Theorem 3 together imply that the operator $F$ determined by formula (14) is an initial operator for $D=D_{1} \ldots D_{m}$ corresponding to the right inverse $R$ of $D$ determined by formula (13).

Suppose we are given an operator $D \in R(X)$ and an initial operator $F^{\prime}$ for $D$ corresponding to an $R \in \mathscr{R}_{D}$. Write

$$
Q(D)=\sum_{k=0}^{N} Q_{k} D^{k}, \quad \text { where } Q_{v}, \ldots, Q_{N-1} \in L(X), Q_{N}=I
$$


We recall (cf. [2]) that and initial value problem for the operator $Q(D)$ is to find all solutions of the equation

$$
Q(D) x=y, \quad y \in X,
$$

satisfying the condition

$$
F D^{k} x=y_{k}, \quad y_{k} \in Z_{D} \quad(k=0,1, \ldots, N-1) .
$$

This problem is said to be well-posed if it has a unique solution for every $y \in X, y_{0}, \ldots, y_{N-1} \in Z_{D}$. This means that a well-posed homogeneous initial value problem has only zero as a solution. By Corollary 3.1 of [2] an initial value problem (13)-(14) is well posed if -1 is not an eigenvalue of the operator

$$
\hat{Q}=\sum_{k=0}^{N-1} Q_{k} R^{N-k}
$$

Indeed, in this case the operator $I+\hat{Q}$ is invertible and a unique solution of the problem under consideration is

$$
x=R^{N}(I+\hat{Q})^{-1}\left[y-\sum_{m=0}^{N-1}\left(\sum_{k=0}^{m} Q_{k} R^{m-k}\right) y_{m}\right]+\sum_{k=0}^{N-1} R^{k} y_{k}
$$

In particular, if $Q_{0}=Q_{1}=\ldots=Q_{N-1}=0$, then $\hat{Q}=0$. We therefore conclude that in this case problem (1.18)-(1.19) is well-posed and its solution is

$$
x=R^{N} y+\sum_{k=0}^{N-1} R^{k} y_{k}
$$

The Corollary 3.1 of [2], mentioned above, can easily be generalized to a larger class of operators. Namely, we have

THEOREM 5. Suppose that the operator $D \in R(X)$, that $F$ is an initial operator for $D$ corresponding to an $R \in \mathscr{R}_{D}$ and that the operator $Q(D)$ is defined by formula (1.17). If -1 is not an eigenvalue of the operator $\hat{Q}$ defined by formula (20), then the initial value problem

$$
\begin{gathered}
Q(D) D^{M} x=y, \quad y \in X \quad(M \geqslant 0), \\
F D^{k} x=y_{k}, \quad y_{k} \in Z_{D} \quad(k=0,1, \ldots, N+M-1)
\end{gathered}
$$

is well-posed and its unique solution is of the form

$$
x=R^{N+M}(I+\hat{Q})^{-1}\left[y-\sum_{m=0}^{N-1}\left(\sum_{k=0}^{m} Q_{k} R^{m-k}\right) y_{M+m}\right]+\sum_{m=0}^{N+M-1} R^{m} y_{m}
$$

Proof. Put $u=D^{M} x$. Then we have

$$
F^{\prime} D^{k} u=F D^{M+k} x=y_{k+M} \quad \text { tor } k=0,1, \ldots, N-1 \text {. }
$$


Therefore we can rewrite problem (23)-(24) as follows:

$$
\begin{aligned}
Q(D) u & =y, \\
F D^{k} u=y_{k+M} \quad(k & =0,1, \ldots, N-1) .
\end{aligned}
$$

According to Corollary 3.1 of [2] and formula (21) this problem is well-posed and has a unique solution

$$
u=R^{N}(I+\hat{Q})^{-1}\left[y-\sum_{m=0}^{N-1}\left(\sum_{k=0}^{m} Q_{k} R^{m-k}\right) y_{M+m}\right]+\sum_{k=0}^{N-1} R^{k} y_{k+M} .
$$

Having already determined $u=D^{M} x$, we consider the following initial value problem:

$$
D^{M} x=u, \quad F D^{k} x=y_{k} \quad(k=0,1, \ldots, M-1) .
$$

This problem is also well-posed and formula (22) implies that its unique solution is of the form:

$$
x=R^{M} u+\sum_{m=0}^{M-1} R^{m} y_{m}
$$

This and formula (28) together imply that problem (23)-(24) is wellposed and has a unique solution

$$
\begin{aligned}
& x= R^{M} u+\sum_{m=0}^{M-1} R^{m} y_{m} \\
&=R^{M}\left\{R^{N}(I+\hat{Q})^{-1}\left[y-\sum_{m=0}^{N-1}\left(\sum_{k=0}^{m} Q_{k} R^{m-k}\right) y_{M+m}\right]+\right. \\
&\left.+\sum_{k=0}^{N-1} R^{k} y_{k+M}\right\}+\sum_{m=0}^{M-1} R^{m} y_{m} \\
&=R^{N+M}(I+\hat{Q})^{-1}\left[y-\sum_{m=0}^{N-1}\left(\sum_{k=0}^{m} Q_{k} R^{m-k}\right) y_{M+m}\right]+ \\
& \quad+\sum_{k=0}^{N-1} R^{k+M} y_{k+M}+\sum_{m=0}^{M-1} R^{m} y_{m} \\
&=R^{N+M}(I+\hat{Q})^{-1}\left[y-\sum_{m=0}^{N-1}\left(\sum_{k=0}^{m} Q_{k} R^{m-k}\right) y_{M+m}\right]+ \\
& \quad+\sum_{m=0}^{N+M-1} R^{m} y_{m},
\end{aligned}
$$

which was to be proved. 
A similar statement is also true for the operator $D^{M} Q(D)$.

In [5] we have shown some applications of Theorems 3 and 4 to onedimensional multi-waves operators, i.e., to operators of the form

$$
D=D_{1} \ldots D_{m},
$$

where

$$
D_{j}=\frac{\partial^{2}}{\partial s^{2}}-\frac{1}{c_{j}^{2}} \frac{\partial^{2}}{\partial t^{2}} \quad(j=1, \ldots, m)
$$

and $c_{j}$ are given constant coefficients. Namely, if we choose operators $A_{1}, \ldots, A_{m}$ in formulae (13) and (14) in an appropriate way, we obtain various probleme for the operator $D$, such the generalized Cauchy problem, the Darboux-Picard problem, etc.

Here we show only that this method can be used for equations with variable coefficients. It is enough to consider one operator of the form

$$
\hat{D}=\frac{\partial^{2}}{\partial \xi^{2}}-b(\tau) \frac{\partial^{2}}{\partial \tau^{2}},
$$

where the function $1 / b(\tau)$ is measurable and locally integrable, the function

$$
a(\tau)=\int \frac{b(\theta)}{b \theta}
$$

is differentiable at each point and, moreover, the function

$$
a(\tau)=a(\tau) \tau
$$

is one-to-one.

Write

$$
s=\xi-a(\tau) \tau, \quad t=\xi+a(\tau) \tau .
$$

It is easy to check that this change of variables permits us to rewrite the operator $\hat{D}$ as follows:

where

$$
\begin{aligned}
\hat{D} & =\frac{\partial^{2}}{\partial \xi^{2}}-b(\tau) \frac{\partial^{2}}{\partial \tau^{2}} \\
& =4 \frac{\partial^{2}}{\partial t \partial s}+\frac{1}{b\left[\alpha^{-1}\left(\frac{t-s}{2}\right)\right]}\left(\frac{\partial}{\partial t}-\frac{\partial}{\partial s}\right)=4(D+H),
\end{aligned}
$$

$$
D=\frac{\partial^{2}}{\partial t \partial s}, \quad H=\frac{1}{4 b\left[a^{-1}\left(\frac{t-s}{2}\right)\right]}\left(\frac{\partial}{\partial t}-\frac{\partial}{\partial s}\right)
$$


The operator $D$ is obviously right invertible and has a right inverse, for instance of the form

$$
(R x)(t, s)=\int_{0}^{t} \int_{0}^{s} x(\xi, \eta) d \eta d \xi
$$

It is not difficult to verify that under our assumptions the operator $I+R H$ is invertible (in the space of continuous functions). Thus the operator $\hat{D}=4(D+H)$ has a right inverse

$$
\hat{R}=\frac{1}{4}(I+R H)^{-1} R \text {. }
$$

Indeed,

$$
\begin{aligned}
\hat{D} \hat{R}=4(D+H) \frac{1}{4}(I+R H)^{-1} R & =(D+D R H)(I+R H)^{-1} R \\
& =D(I+R H)(I+R H)^{-1} R=D R=I .
\end{aligned}
$$

Thus we can apply all the results of papers [2], [5] and the present paper to operators $\hat{D}$ of the form (31).

In a similar way we can consider the case where the coefficient of the operator $\hat{D}$ defined by formula (31) depends on the variable $\xi$ :

$$
b=b(\xi) \text {. }
$$

\section{References}

[1] R. Arens Dense Limit Rings, Michigan Math. J. 5 (1958), p. 159-182.

[2] D. Przoworska-Rolowicz, Algebraic theory of right invertible operators, Studia Math. 48 (1973), p. 129-144.

[3] - Pseudocategories; Para-algebras and Perturbations of linear operators, Preprint No. 60, Institute of Mathematics Polish Academy of Sciences, Warszawa 1973.

[4] - Concerning left invertible operators, Bull. Acad. Polon. Sci. 20 (1972), p. 837-839.

[5] - A characterization of admissible initial value problems for one-dimensional multi-waves equations (Polish), Biuletyn WAT 7 (263) (1974), p. 23-30.

INSTYTUT MATEMATYCZNY PAN

INSTITUTE OF MATHEMATICS OF THE POLISH ACADEMY OF SCIENCES

Reģu par la Rédaction le 10. 7. 1974
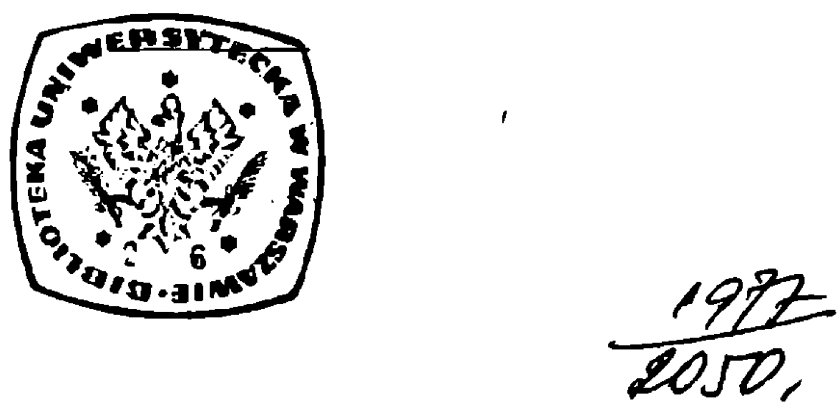


\section{LIVRES PUBLIÉS PAR L'INSTITUT MATHEMATIQUE DE L'ACADÉMIE POLONAISE DES SCIENCES}

S. B anach, Oeuvres, Vol. I, 1967, p. 381.

S. Mazurkiewicz, Travaux de topologie ot ses applications, 1969, p. 380 .

W. Sierpiński, Oeurres choisies, tome I, 1974, p. 300; tome II, 1975, p. 780; tome III, 1976, p. 688.

\section{MONOGRAFIE MATEMATYCZNE}

41. H. Rasiowa and R. Sikorski, The mathematics of metamathematics, 3-ème éd., corrigée, 1970, p. 520.

42. W. Sierpiński, Elementary theory of numbers, 1964, p. 480.

43. J. Szarski, Differential inequalities, 2-ème éd., 1967, p. 256.

44. K. Borsuk, Theory of retracts, 1967, p. 251.

45. K. Maurin, Methods of Hilbert spaces, 2-ème éd., 1972, p. 552.

47. D. Przeworska-Rolewicz and S. Rolewicz, Equations in linear spaces, 1968, p. 380.

50. K. Borsuk, Multidimensional analytic geometry, 1969, p. 443.

5I. R. Sikorski. Advanced calculus. Functions of several variables, 1969, p. 460.

52. W. Slebodziński, Exterior forms and their applications, 1970, p. 427.

53. M. Krzyżański, Partial differential equations of second order I, 1971, p. 562.

54. M. Krzyżański, Partial differential equations of second order II, 1971, p. 407.

57. W. Narkiewicz, Elementary and analytic theory of algebraic numbers, 1974, p. 630 .

58. C. Bessaga and A. Pelczynski, Selected topics in infinite-dimmensional topology, 1975, p. 353.

59. K. Borsuk, Theory of shape, 1975, p. 384 .

60. R. Engelking, General topology, 1977, sous presse.

\section{Nouvelle série:}

\section{BANACH CENTER PUBLCIATIONS}

1. Mathematical control theory, 1976, p. 166.

2. Mathematical foundations of computer sciences (sous presse).

\section{LES DERNIERS FASCICULES DES DISSERTATIONES MATHEMATICAE}

CXXXVI. J. Y. Girard, Three valued logio and cut-elimination: The actual meaning of Takeuti's conjecture, 1976, p. 1-50.

CXXXVII. K. Hofman, A. Stralka, The algebraic theory of oompact Lawson semilattices. Applications of Galois connections to compact semilattices, 1976 , p. $1-58$.

CXXXVIII. Sam B. Nadler, Jr., Arcwise accessibility in hyperspaces, 1976, p. 1-33.

CXXXIX. T. Yoshimoto, Induced contraction semigroups and random ergodio theorems, 1876, p. 1-45. 